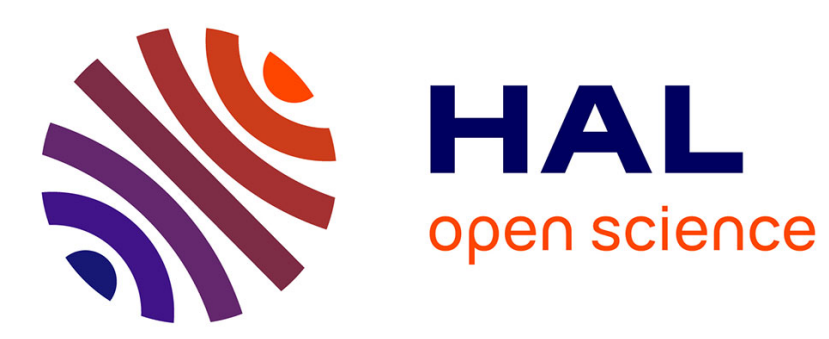

\title{
Parlement et contraintes discursives
}

\author{
Baudouin Dupret, Enrique Klaus, Jean-Noël Ferrié
}

\section{To cite this version:}

Baudouin Dupret, Enrique Klaus, Jean-Noël Ferrié. Parlement et contraintes discursives: Analyse d'un site dialogique. Réseaux: communication, technologie, société, 2008, 148-149, pp.369-404. halshs-00311642

\section{HAL Id: halshs-00311642 https://shs.hal.science/halshs-00311642}

Submitted on 20 Aug 2008

HAL is a multi-disciplinary open access archive for the deposit and dissemination of scientific research documents, whether they are published or not. The documents may come from teaching and research institutions in France or abroad, or from public or private research centers.
L'archive ouverte pluridisciplinaire HAL, est destinée au dépôt et à la diffusion de documents scientifiques de niveau recherche, publiés ou non, émanant des établissements d'enseignement et de recherche français ou étrangers, des laboratoires publics ou privés. 
Page $1 / 3$ 
PARLEMENT ET CONTRAINTES DISCURSIVES

Analyse d'un site dialogique

Baudouin DUPRET

Enrique KLAUS

Jean-Noël FERRIE 
Dans cet article, nous nous proposons de rendre compte des développements parlementaires d'une affaire égyptienne. Nous nous intéressons à l'une des chambres du Parlement, l'Assemblée du Peuple, en tant qu'elle est à la fois un maillon d'un réseau dialogique unissant les médias, les institutions politiques et leurs différents publics et un lieu de débat à part entière, ce que nous appelons un "site dialogique ». Le débat parlementaire est une activité institutionnelle dotée de ses propres finalités pratiques qui répond à une logique pour partie spécifique et qui est encadrée par des procédures particulières contraintes discursivement et procéduralement. En ce sens, le débat à l'Assemblée du Peuple transforme séquentiellement l'affaire médiatique en un objet parlementaire, c'est-à-dire marqué par le jeu de la représentation politique (élections, majorité, opposition) et doublement orienté vers l'intérieur et l'extérieur de l'enceinte. L'étude détaillée du déploiement du débat à propos d'un scandale permet de démonter, instant après instant, pièce par pièce, les ressorts propres à la pratique politique située des membres d'une assemblée spécifique sur une question touchant à la moralité publique. Elle montre comment les préoccupations morales sont insérées dans la dynamique de l'ordre parlementaire et transformées en un jeu relativement limité de pertinences et de contrastes fondés, non sur la nature morale intrinsèque de la question débattue ou sur la spécificité culturelle du pays où l'affaire se déroule, mais sur la nature de l'activité parlementaire ${ }^{1}$.

\footnotetext{
${ }^{1}$ Tous nos remerciements à Alain Bovet, Dominique Linhardt et Louis Quéré pour leurs commentaires précieux sur une version antérieure de cet article.
} 
De manière générale, les débats parlementaires demeurent un objet fort peu étudié, sinon, récemment, dans une perspective se rattachant au mouvement de l'analyse critique de discours (critical discourse analysis) ${ }^{2}$. Nous menons, pour notre part, une démarche praxéologique. Dans cette perspective, la séance parlementaire se présente comme un instant particulier du débat public, du fait qu'elle s'inscrit dans un contexte institutionnel spécifique orienté vers des fins pratiques déterminées (e.g. vote des lois, questions au gouvernement, discussion et approbation du budget) et organisé selon des règles procédurales précises (Règlement intérieur). L'institution parlementaire, souvent considérée comme le lieu par excellence de la délibération politique, apparaît ainsi dans la forme d'un site dialogique ${ }^{3}$. Il s'agit d'un site, en ce sens que les activités s'y inscrivent dans un contexte unique et précis et qu'elles y sont contraintes du fait même de cette insertion contextuelle spécifique. Ce site est dialogique, dans la mesure où, au sein d'une assemblée parlementaire, productrice d'un ordre social endogène, différentes questions peuvent être débattues et mettre aux prises une grande variété de conceptions trouvant à s'énoncer et à se confronter dans un cadre institutionnel, procédural et argumentatif partagé, sous la forme généralement d'échanges entre participants autorisés. Le débat sur le site dialogique du Parlement s'inscrit, par ailleurs, dans le déploiement d'un réseau, lui aussi dialogique, fait d'occurrences distantes dans le temps et dans l'espace, médiatisées et connectées entre elles thématiquement, interactivement et argumentativement ${ }^{4}$.

Dans la présente étude, nous nous intéressons à l'affaire «Fârûq Husnî et le combat du voile $»^{5}$ telle qu'elle a été traitée sur le site dialogique de l'Assemblée du Peuple. La question du voile, qui avait défrayé plusieurs fois la chronique égyptienne des mois précédents, est relancée par la publication, le 16 novembre 2006, d'un article intitulé : «Husnî : le voile est un retour en

\footnotetext{
${ }^{2}$ Cf. e.g. BAYLEY, 2004. Pour une évaluation critique de ce mouvement, en particulier dans sa conception du contexte de l'interaction parlementaire, cf. DUPRET \& FERRIE, 2008.

${ }^{3} \mathrm{Cf}$. DUPRET, BELHADJ, FERRIE, 2007.

${ }^{4}$ L'analyse de conversation envisage les échanges discursifs au sein d'une conversation comme autant de tours de parole dont il convient d'analyser l'articulation les uns aux autres, de manière séquentielle. Cette notion de tours de parole s'entend, dans son sens le plus ordinaire, comme un énoncé formulé par une intervenant coprésent et répondant à un énoncé formulé par l'intervenant précédent. L'idée d'un réseau dialogique (NEKVAPIL \& LEUDAR, 2002 ; LEUDAR et al., 2004 ; LEUDAR \& NEKVAPIL, 2007) consiste à étendre ce système des tours de parole au-delà de la situation de coprésence, à l'ensemble des échanges médiatisés. On remarquera les affinités avec certains travaux sur la circulation des récits médiatiques en infocom (cf. e.g. différents articles parus précédemment dans Réseaux).

${ }^{5}$ Selon la formule utilisée en manchette du journal al-Misrî al-yawm, 18 novembre 2006.
} 
arrière ». L'affaire prend de l'ampleur et la forme d'une confrontation politique entre le gouvernement et les Frères musulmans, principale force de l'opposition parlementaire. Les protagonistes s'engagent alors dans une tentative d'extension du réseau et cherchent à convoquer les instances de décision, au nombre desquelles le Parlement ${ }^{6}$.

Notre analyse du débat parlementaire s'inscrit dans un projet plus vaste d'étude praxéologique de l'activité parlementaire entendue comme parole institutionnelle inscrite dans le cadre plus large du débat public. Ce faisant, nous ajoutons une nouvelle instance à l'éventail d'études menées en analyse de conversation sur le contexte institutionnel ${ }^{7}$. Après avoir présenté la séquence parlementaire, il s'agira ici de détailler comment la " conversation » parlementaire acquiert une dimension institutionnelle, notamment par le dispositif de tours de parole qui en émerge ${ }^{8}$. Nous nous intéresserons également à certaines des entorses qui lui sont faites: les applaudissements et les interventions, afin de montrer comment le caractère institutionnel et institutionnalisé du débat parlementaire constitue, de différentes façons, à la fois une contrainte et une ressource pour les participants. Enfin, nous serons amenés à nous intéresser à la mobilisation des pertinences et des audiences qui s'imposent comme ressources d'alignement discursif, permettant de dépasser la contrainte institutionnelle qui pèse sur un discours devant se déployer dans un site dialogique.

Cette étude se fonde sur les retranscriptions verbatim officielles disponibles sur le site de l'Assemblée du Peuple (http://www.parliament.gov.eg). Il ne s'agit donc pas de l'enregistrement audio ou audiovisuel du débat à partir duquel nous aurions pu mener une analyse de conversation au sens strict. Notre matériau est fait de la production textuelle et archivistique des services du Parlement égyptien, constituée aux fins pratiques et bureaucratiques de l'activité parlementaire. Il traduit un souci d'exactitude, selon des règles développées de manière endogène par le secrétariat du Parlement, mais aussi

\footnotetext{
${ }^{6}$ Sur l'affaire Fârûq Husnî en tant que réseau dialogique, nous renvoyons à deux articles formant, avec celui-ci, une sorte de triptyque: KLAUS, DUPRET, FERRIE, 2008; DUPRET, KLAUS, FERRIE, 2008.

${ }^{7}$ Pour une présentation synthétique, critique et constructive de la recherche sur la parole institutionnelle (institutional talk), cf. la thèse d'Alain Bovet (BOVET, 2007), et particulièrement les pages 292-306. Le programme de l'institutional talk a déjà étudié une série de contextes, tels que les tribunaux, l'interview journalistique, la classe d'école l'activité policière, le milieu hospitalier. Cf. e.g.. ATKINSON \& DREW, 1979; DUPRET, 2006; GREATBATCH, 1988; HERITAGE \& GREATBATCH, 1991 ; MCHOUL, 1978 ; MEHAN, 1985; MEEHAN, 1997; HEATH, 1986.

${ }^{8}$ Cf. e.g. HERITAGE, 1998
} 
dans le respect d'une procédure pouvant justifier un travail d'édition (ainsi en va-t-il des élisions volontaires des propos à caractère injurieux ou jugés tels, de l'utilisation de caractères gras pour les citations coraniques ou la description d'actions spécifiques, ou encore des formules désignant les différents orateurs). Ces documents sont toutefois suffisamment détaillés (registre langagier, erreurs syntaxiques, répétitions, interruptions, applaudissements, etc.) que pour attester de leur caractère fidèle à l'original interactionnel. Sans pouvoir prétendre atteindre l'idéal de pureté de l'analyse de conversation, le matériau présente une fiabilité qui nous a semblé d'autant plus suffisante qu'il constitue la base des usages pouvant en être faits dans les développements ultérieurs de la controverse.

\section{La séquence parlementaire}

Dans l'affaire "Fârûq Husnî et le combat du voile ", le débat intervient à l'occasion de la première session tenue par l'Assemblée du Peuple après les vacances parlementaires. La veille, le 19 novembre 2006, le Président de la République avait prononcé son discours traditionnel devant les députés, auquel le ministre de la Culture n'avait d'ailleurs pas assisté. Le débat se déploie de manière séquentielle, dans une succession d'interventions contiguës, d'interruptions interactives et d'alignements pragmatiques. Quelques modalités spécifiques d'intervention dans le contexte parlementaire semblent émerger. Tout d'abord, les interventions présidentielles, qui se déclinent en différents types : la lecture de l'ordre du jour, les orientations procédurales, l'attribution des tours de parole, les questions formulées aux parlementaires à la suite ou dans le cours de leurs interventions, les réponses éventuelles aux questions qui lui sont posées incidemment, les digressions pédagogiques par lesquelles il explique tel aspect de la loi ou du fonctionnement de l'Assemblée, les " réparations »" aux entorses faites au fonctionnement «normal» de celle-ci, les interruptions portant sur des points d'ordre ou sur certaines questions de fond. Deuxièmement, on remarque les tours de parole légitimes des parlementaires ou du ministre des Affaires juridiques et parlementaires, que le président invite formellement. Troisièmement, les interruptions à ces tours de parole, qui sont toujours illégitimes (sauf dans le cas du président), prennent une forme discursive ou des formes gestuelles et sonores. Ces

\footnotetext{
${ }^{9}$ La notion de réparation s'entend ici au sens que lui confère l'analyse de conversation. Cf. e.g. SCHEGLOFF 2007.
} 
interruptions, quatrièmement, peuvent être ignorées ou prises en compte par l'orateur légitime. Cinquièmement, des applaudissements peuvent ponctuer certains propos, en cours ou en fin de tour de parole. Enfin, le président est amené à soumettre à l'approbation de l'Assemblée le renvoi du débat sur la déclaration du ministre à une commission conjointe.

Le compte-rendu de la séance de l'Assemblée du 20 novembre commence par les mentions légales: "L'Assemblée s'est réunie à 11 heures 15 du matin, sous la présidence de Monsieur le Professeur Docteur Ahmad Fathî Surûr, président de l'Assemblée ». Suit la mention des membres de l'Assemblée excusés et absents et celle des ministres présents, faisant apparaître par défaut l'absence des autres et, en particulier, de Fârûq Husnî ${ }^{10}$. Le président Surûr commence par l'annonce officielle de l'ouverture de la séance :

Extrait 1 : séance parlementaire du 20 novembre 2006 (retranscription verbatim)

Le président de l'Assemblée

1 Au nom de Dieu, au nom du peuple, je [déclare] ouverte

2 la session. Au nom de Dieu, le Clément, le Miséricordieux.

3 «Dis : Agissez! Dieu verra vos actions ainsi que le prophète et les

4 croyants " (Vérité du Dieu Puissant) [Coran 9, 105]

Cette formulation présente une double caractéristique : d'une part, elle place la session parlementaire sous l'égide de Dieu et du peuple, là où seule la mention du peuple aurait suffi juridiquement, ce qui a pour effet immédiat de poser la légitimité constituante des registres religieux et démocratique, à moins qu'il ne s'agisse tout simplement de correction civile et procédurale ; d'autre part, elle réalise performativement une tâche institutionnelle, l'inauguration, marquant par là-même la nature spécifique des activités qui prendront place à l'intérieur du cadre procédural ainsi constitué. La citation coranique, en gras, est récurrente dans les transcriptions des sessions parlementaires égyptiennes. Elle inscrit dès lors cette action dans la routine religieusement référencée des activités de l'Assemblée du Peuple.

Après avoir déclaré la session ouverte, le président de l'Assemblée engage la première activité, à savoir la lecture des missives adressées au Parlement.

\footnotetext{
${ }^{10}$ Cette absence traduit, entre autres choses, la faible responsabilité des ministres devant le Parlement, dans le système égyptien.
} 
Ensuite, il procède à l'examen des communications urgentes qui, toutes, portent sur l'affaire "Fârûq Husnî et le combat du voile ». Ce faisant, le président accomplit une deuxième tâche performative, à savoir inscrire le débat qui va suivre dans le registre pratique spécifique de la discussion publique. Ce n'est donc pas de la fonction législative du Parlement qu'il s'agit ici, mais de sa fonction d'instance de discussion des questions d'intérêt général

Extrait 2 : séance parlementaire du 20 novembre 2006 (retranscription verbatim)

5 (En premier lieu), les missives

Le président de l'Assemblée

$6 \quad$ Lisons les missives (les missives sont lues)

7 Décisions de Monsieur le Président de la République concernant

8 l'aval sur les accords internationaux suivants :

$9 \quad[\ldots]$

10 (En deuxième lieu), mention faite par le prof. Docteur président de

11 l'Assemblée des demandes de communication urgente présentées par

12 quelques uns de Messieurs les députés concernant les déclarations

13 faites par Monsieur le ministre de la Culture à la presse et aux médias

14 à propos du voile (hijâb), le transfert devant la commission de la

15 culture, des médias et du tourisme et le discours de Monsieur le

16 ministre qu'il a envoyé pour mettre au clair ce qui a été publié.

Cet extrait montre comment ce site dialogique qu'est l'Assemblée du Peuple s'articule au réseau dialogique de l'affaire initiée dans la presse. En faisant mention des demandes de communication urgente de plusieurs députés, le président atteste de la pertinence parlementaire d'une question trouvant son origine en-dehors de cette enceinte. En lisant la lettre envoyée par le ministre de la Culture à l'Assemblée, le président incorpore dans l'ordre du jour des activités parlementaires, de manière intertextuelle, les déclarations, les réactions qu'elles ont suscitées et la réponse à ces réactions.

Extrait 3 : séance parlementaire du 20 novembre 2006 (retranscription verbatim)

Le président de l'Assemblée

33 Je vous lis à haute voix le discours de Monsieur le ministre de la

34 Culture : "Monsieur le Professeur Ahmad Fathî Surûr, président

35 de l'Assemblée. Salutations distinguées. 
36 La presse et les médias ont relayé les points de vue variés de 37 nombre de Messieurs les députés de l'Assemblée du Peuple 38 commentant un point de vue qui m'est personnel concernant le 39 costume de la femme égyptienne et qui se résume à des 40 objections artistiques et formelles. Outre le fait qu'il y a eu une 41 ambiguité dans la compréhension de ce que j'ai déclaré, je ne 42 cherche pas à savoir si ce quiproquo était spontané ou 43 intentionnel ('afwiyya aw 'amdiyya). Mais, sauf mon respect 44 pour l'Assemblée du Peuple et l'ensemble de ses membres, 45 j'[aimerais] clarifier ce qui suit : [...]

Cette intertextualité, spécifique à tout réseau dialogique, qui consiste à indexer le débat en cours sur des éléments extérieurs et antérieurs à l'interaction sur le site dialogique, se retrouve à différents moments de la séance. Ainsi, l'extrait suivant :

Extrait 4 : séance parlementaire du 20 novembre 2006 (retranscription verbatim)

Le président de l'Assemblée

374 Je prie Monsieur l'honorable député Bâha' al-Dîn Sayyid

375 'Atiyya de [nous faire part de] ses commentaires.

M. l'hon. député B.D. Sayyid 'Atiyya

376 Au nom de Dieu, le Clément, le Miséricordieux.

377 Monsieur le président de l'Assemblée, frères et

378 sœurs députés de l'honorable Assemblée.

379 Il apparaît bien dans les déclarations du ministre égyptien de la

380 Culture que le voile est un retour en arrière (ridda ila al-warâ'),

381 qu'il est arriération et qu'il [le ministre] n'est pas convaincu par

382 cette affaire. Et moi, je dis au ministre de la Culture, qui êtes-

383 vous pour tenir ces propos et dire que le voile est un retour en

384 arrière; alors que Dieu - qu'Il soit béni et exalté - nous a

385 commandé dans son cher livre :

386 Au nom de Dieu le Clément, le Miséricordieux, "Ô Prophète,

387 Dis à tes épouses, à tes filles et aux femmes des croyants de

388 rabattre sur elles leurs voiles afin de se faire connaitre et de ne

389 pas être offensées - Dieu est celui qui pardonne, Il est

390 Miséricordieux ». Parole du Dieu Puissant. [Coran 33, 59] [...]

398 Ces jours-ci, Fârûq Husnî arrive et accuse le voile d'être un

399 retour en arrière. Mais qui est ce ministre ?! Moi, j'exige, au nom

400 du peuple, j'exige que Monsieur le Président de la République, 
401 personnellement, démissionne ce ministre de toute urgence. S'il

402 n'a pas pu [le faire] et s'il n'a pas fait... (applaudissements) ..

Cette intervention est intertextuellement riche. Elle incorpore la voix de l'orateur qui non seulement prend la parole mais interpelle directement ses collègues et le ministre de la Culture. Elle mobilise la voix du ministre, quand elle en cite les propos. Elle adresse la parole directement au président de l'Assemblée, le convoquant ainsi dans la trame discursive. Elle en appelle aussi à l'Assemblée et à ses membres, sur le double registre institutionnel et religieux. Elle procède de plus à deux citations du Coran, intégrant de la sorte la voix de Dieu après en avoir appelé aux collègues en leur qualité de coreligionnaires. Enfin, elle se pose " au nom du peuple» et s'adresse au Président de la République. Ce ne sont donc pas moins de sept actants, au sens sémiotique, qui contribuent à donner à cette intervention sa force rhétorique. En outre, le jeu des indexicaux accentue le contraste entre les propos du ministre, cités à la forme indirecte, et Dieu, cité dans le texte (dont les paroles sont formulées à l'impératif et indexent une série d'autres figures, tels le Prophète, les croyants et leurs femmes). Ce contraste suscite des applaudissements (cf. infra). Enfin, l'orateur s'adresse, selon des formes différentes, à deux instances absentes: à la forme directe au ministre, interpellé lui-même à la deuxième personne, ce qui a pour effet d'accentuer l'effet d'injonction ; à la troisième personne au Président Mubârak, ce qui dénote davantage une requête.

\section{Tours de parole et rôle présidentiel}

La prise de parole, au sein d'une chambre parlementaire comme l'Assemblée du Peuple, est régie par un Règlement intérieur qui en détaille les modalités. Un ordre du jour est établi préalablement à la tenue de la séance, sous la responsabilité du président, et les membres de l'Assemblée ont l'occasion de s'y inscrire selon des procédures précises. La « demande de communication urgente " appartient à ces formes préalables de sollicitation de la parole. On observe ici comment les membres d'une institution délibérative s'orientent vers les règles procédurales propres à celle-ci. On peut appeler ce mécanisme " solidarité procédurale sans consensus politique », par quoi l'on vise l'acceptation commune des règles formelles d'un jeu institutionnel sans accord nécessaire sur les finalités politiques de leur usage. En demandant qu'il soit pris connaissance d'un fait et en justifiant que cela se fasse de manière urgente, dérogeant de la sorte aux délais normalement établis, les 
parlementaires reconnaissent la légitimité du Parlement et confèrent à son Règlement intérieur une valeur de ressors d'ordre, s'étendant in fine au-delà du simple respect de la procédure pour elle-même, puisqu'il permet une hiérarchisation des événements extérieurs.

Le rôle pivotal du président de la chambre ${ }^{11}$ apparait également d'emblée, au travers de sa fonction de distributeur et de régulateur de la prise de parole et du pouvoir d'influence qui en découle.

\section{Extrait 5 : séance parlementaire du 20 novembre 2006 (retranscription verbatim)}

Le président de l'Assemblée

408 J'ai [déjà] dit que le débat serait limité et que tous les députés ne

409 pourront pas parler sur ce sujet.

410 (À présent, Monsieur l'honorable député Mohammad 'Âmir

411 Hilmî s'avance vers la tribune en demandant la parole)

Le président de l'Assemblée

412 Je dis à Monsieur le député Muhammad 'Amir Hilmî de retourner à

413 sa place.

414 (Voix de Monsieur l'honorable député Muhammad 'Âmir

415 Hilmî : j'ai un bulletin urgent avant ces intervenants)

Le président de l'Assemblée

416 Si vous avez un bulletin demandant une communication urgente,

417 attendez que votre tour de parole vienne. Mais si vous venez à la

418 tribune pour prendre la parole, alors non. Et celui qui vient à la

419 tribune pour la parole, il ne la prendra pas. Il y a un système, il y a des

420 traditions. Je prie Monsieur l'honorable député 'Âdil Sha'lân de [nous

421 faire part de] ses commentaires.

Par ailleurs, outre qu'il appartient au président de juger du caractère justifié des demandes de communication urgente, il lui revient aussi, à l'aide des pouvoirs que le Règlement intérieur lui confère, d'orienter la procédure suivie pour le traitement des questions mises à l'ordre du jour et ainsi d'en assurer l'apaisement au moins provisoire (renvoi en commission) ou l'exacerbation (traitement à chaud). Tout en n'intervenant que rarement sur le fond, le président joue donc un rôle déterminant dans l'issue que trouve toute question soulevée à l'Assemblée.

${ }^{11}$ DUPRET, BELHADJ, FERRIE, 2007. 


\title{
Extrait 6 : séance parlementaire du 20 novembre 2006 (retranscription
} verbatim)

\author{
225 À présent, êtes-vous d'accord pour déférer ce sujet devant la \\ 226 commission de la culture, des médias et du tourisme [ainsi que] \\ 227 la commission des affaires religieuses et sociales et des waqf \\ 228 pour préparer un rapport qui sera présenté à l'Assemblée ? \\ 229 (Approbation) \\ 230 Alors, le sujet sera déféré devant la commission de la culture, \\ 231 des médias et du tourisme [ainsi que] la commission des affaires \\ 232 religieuses et sociales et des waqf. À présent, je prie les deux \\ 233 commissions [de bien vouloir] se réunir et écouter le ministre, \\ 234 puis de produire un rapport qui sera à nouveau présenté devant \\ 235 l'Assemblée. \\ 236 À présent, entamons les débats sur le calendrier des travaux \\ 237 [parlementaires] [...]
}

Si l'on s'attarde quelque peu sur la prise de parole du président, on constate comment il est amené à jouer avec les dispositions réglementaires définissant son rôle. L'article 6 du Règlement intérieur de l'Assemblée du Peuple dispose que :

Le président de l'Assemblée est celui qui la représente et parle en son nom conformément à sa volonté. Il préserve sa sécurité, sa discipline, sa dignité et la dignité de ses membres et veille, de manière générale, au bon déroulement des travaux de l'Assemblée. Le Président ouvre les séances, les préside, annonce leur conclusion, les discipline, dirige les débats, donne [les tours de] parole, définit le sujet [des travaux] et rappelle à l'attention de l'orateur de s'en tenir aux limites du débat. Il doit clarifier ou demander de clarifier les questions qu'il considère obscures. Il décide de tout ce qui doit [faire l'objet] d'un vote. Il est [également] celui qui annonce les décisions prises par l'Assemblée. Le président a le droit de donner son avis en participant au débat sur n'importe quelle question proposée, auquel cas il doit se dégager de la présidence de l'Assemblée et ne pas rester à la barre du président jusqu'à ce que la discussion à laquelle il a pris part se termine.

Une des prérogatives spécifiques du président de l'Assemblée tient à ce qu'il peut s'auto-attribuer la parole, non seulement pour assurer le passage harmonieux des tours ou assurer le bon déroulement de la séance, mais aussi pour " clarifier les questions qu'il considère obscures". En revanche, il lui faut retourner à une qualité de simple parlementaire quand il souhaite 
" donner son avis en participant au débat ». C'est dans une pratique de la norme que la distinction entre les deux situations - clarifier les termes du débat ou y apporter une contribution personnelle - trouve à se négocier. On observe ainsi comment la norme et, en l'espèce, les dispositions du Règlement intérieur, loin d'être une contrainte extérieure s'imposant de manière univoque, sont l'objet de transactions constantes.

Le président est donc le seul membre de l'Assemblée à pouvoir entrer véritablement (et légitimement) en interaction avec les autres participants au débat. Il peut ainsi décider de les corriger :

Extrait 7 séance parlementaire du 22 novembre 2006 (retranscription verbatim) $^{12}$

M.A.F. Mustafâ (M. al-Sahafi)

$10 \quad[\ldots]$ Sans préjudice pour son excellence, c'est lui

11 qui a commencé et nous qui, nous appuyant sur une volonté

12 populaire, avons entrepris de rétorquer à son excellence; votre

13 excellence et [nous autres les membres de] l'Assemblée, nous

14 avons décidé de sa comparution devant la commission jointe ce

15 samedi. Je vois qu'avec ses déclarations...

Le président de l'Assemblée

16 La date de la réunion de la commission jointe n'a pas été fixée et

17 je la fixerai personnellement.

Le président peut également poser des questions aux intervenants (Extrait 6, lignes 256-257) ou répondre à leurs requêtes :

Extrait 8 séance parlementaire du 20 novembre 2006 (retranscription verbatim)

406 voix de l'un de Messieurs les députés: Je veux la parole,

407 Monsieur le président)

Le président de l'Assemblée

408 J'ai [déjà] dit que le débat serait limité et que tous les députés ne

409 pourront pas parler sur ce sujet.

\footnotetext{
${ }^{12}$ Cet extrait est tiré d'une séance parlementaire ultérieure (22 novembre); il s'agit donc d'une résurgence de l'affaire au Parlement, d'une nouvelle instance du réseau dialogique.
} 
Enfin, le président exerce à l'occasion son pouvoir d'interruption des orateurs (Extrait 12, lignes 132-134) ou peut encore faire une digression pédagogique :

\section{Extrait 9 séance parlementaire du 20 novembre 2006 (retranscription verbatim)}

1189 Permettez-moi de vous parler d'une règle de droit dans cette 1190 affaire. Toute personne a le droit public d'expression, mais il y a 1191 une différence entre la personne privée et la personne publique. $1192 \mathrm{La}$ vie privée des personnalités publiques - et par-dessus tout, 1193 des responsables - se confond avec la vie publique au point qu'il 1194 ne peut pas, en toute liberté, faire [des choses] en tant que 1195 personne privée ou émettre des opinions personnelles qu'il tient 1196 en tant que personne privée. [...]

À cette autorité dont est investi le président de l'Assemblée ès qualité s'ajoute, dans le cas qui nous concerne, la personnalité même de celui-ci, qui émerge de manière visible en différents points du débat et qui concourt à renforcer son autorité et sa centralité dans le processus. On notera ainsi les termes avec lesquels les orateurs s'adressent à lui, citant ses qualités de président (de l'Assemblée), de docteur (en droit) et de professeur (d'université) (e.g. Extrait 10, lignes 992-994). Ces termes d'adresse ont parfois une valeur seulement formelle, en introduction ou en conclusion du tour de parole. Parfois, ils servent à rythmer l'intervention. Ils ont aussi, de temps en temps, une valeur majorée. Ainsi, les qualités de juriste éminent habituellement reconnues à Fathî Surûr sont invoquées par un membre de l'Assemblée afin, sans doute, de s'en assurer le soutien, mais aussi peut-être de le mettre en position de ne pouvoir se dédire et, partant, de contraindre son alignement sur des positions critiques à l'égard du ministre. Il s'agit alors d'un cas de "solidarité négative induite ", par quoi on entend que la caution de l'autorité (du président dans ce cas-ci) est obtenue en forçant l'expression d'un consentement par défaut.

Extrait 10 : séance parlementaire du 20 novembre 2006 (retranscription verbatim)

M. l'hon. Député A. 'A Hâshim

989 (applaudissements). Personne, Monsieur le président, personne

990 n'en est davantage gardien [de l'islam] que nous, nous autres ici,

991 [gens] du pays d'al-Azhar et du pays de l'islam, personne ici 
992 n'en est davantage gardien. Vous connaissez le droit et vous êtes

993 plus savant quant à ce qui peut œuvrer à établir la sécurité et

994 à stabiliser les concepts religieux, alors que notre Communauté

995 [islamique] vit à présent une période de discrédit qu'elle n'a pas

996 vécu précédemment [...]

\section{Interruptions et applaudissements}

Dans le contexte procéduralement organisé d'un débat parlementaire, les tours de parole sont distribués par le président entre les membres de l'Assemblée qui en ont fait la demande écrite préalable. Deux types d'exceptions peuvent toutefois survenir. D'une part, les exceptions réglementairement prévues. Ainsi en va-t-il des demandes d'intervention formulées par un certain nombre de personnes intervenant en leur qualité officielle de président de commission parlementaire ou de ministre :

Extrait 11 : séance parlementaire du 20 novembre 2006 (retranscription verbatim)

Le président de l'Assemblée

939 Le sujet est clôturé, mais le président de la Commission des affaires

940 religieuses et sociales et des waqfs demande à parler. Je vous en

941 prie.

M. l'hon. Député A. 'A Hâshim

942 Au nom de Dieu le Clément le Miséricordieux.

943 Merci Monsieur le président.

944 Alors que nous discutons [...]

Le Règlement intérieur de l'Assemblée du Peuple prévoit en effet que les représentants du gouvernement ou les membres du Parlement exerçant une responsabilité peuvent demander à tout instant de prendre la parole et que, dans ce cas, le président la leur accordera. Ici encore, on observe comment les dispositions d'un texte réglementaire constituent des ressources aux mains des participants dont ils usent en fonction du déroulement situé et de la configuration émergente du débat en cours. 
Les interruptions constituent l'autre type d'exception à la distribution ordinaire des tours de parole. Il s'agit dans ce cas d'une technique non réglementaire et illégitime d'intervention. Les interruptions visent, pour l'intervenant, tantôt à formuler des commentaires sur les propos tenus par l'orateur légitime, tantôt à en prolonger les remarques, tantôt encore à en critiquer les positions, tantôt enfin à s'auto-désigner comme orateur suivant. Nous retrouvons plusieurs de ces cas de figure dans l'extrait suivant :

Extrait 12 : séance parlementaire du 20 novembre 2006 (retranscription verbatim)

83 Monsieur l'honorable député, Dr. 'Abd al-Ahmad Jamâl al-Dîn,

84 représentant du groupe parlementaire du PND.

85 Après vous.

86 (Voix de Monsieur le député Muhammad Ahmad Husayn: Je

87 n'imaginais pas que votre excellence lirait à haute voix cette

88 missive et je sais que vous n'en êtes pas convaincu)

Le président de l'Assemblée

89 Professeur Muhammad, il y a des règles à la parole et je vous

90 prie de respecter ces règles et de ne pas parler tant que je ne vous

91 le permets pas.

M. l'hon. Député ‘A. Â Jamâl al-Dîn

92 Monsieur le président, à la vérité, le sujet est irritant pour la

93 société égyptienne. Nous avons bien écouté le discours dans

94 lequel Monsieur le ministre a expliqué le contexte et les tenants

95 de ce qui a trait aux points de vue qu'il a émis et qu'il décrit

96 comme privés. Il a insisté sur le fait qu'il s'agissait d'une

97 opinion personnelle et nous, ici, à l'Assemblée du Peuple, nous

98 respectons cette opinion et nous respectons toutes les opinions

99 qui doivent..

100 (voix de M. le député Muhammad Ahmad Husayn : une opinion

101 contre la religion)

102 (voix de Monsieur le député Karam al-Hifyân : une opinion fausse)

103 Notre devoir, ici, à l'Assemblée du Peuple, alors que nous

104 préconisons l'opinion et l'opinion inverse et que nous respectons

105 l'ensemble des opinions [...]

112 C'est pourquoi, je prie Monsieur l'honorable président et les

113 honorables frères et sœurs députés à l'Assemblée du Peuple de

114 ne pas donner au sujet plus que ce qu'il mérite. Parce que nous

115 avons des sujets d'une extrême importance... 
116 (Voix de Monsieur le député Dr. 'Abd al-Hâfiz Hîrdî :

117 Défendez-vous les erreurs ?!)

M. l'hon. Député 'A. Â Jamâl al-Dîn

118 Il n'est pas sensé que l'Assemblée du Peuple donne à ce sujet

119 une grande ampleur. Nous, nous avons de nombreux problèmes

120 que nous a soumis le président Muhammad Husnî Mubârak.

121 Cher Monsieur, mon propos n'a rien à voir avec cela. Et nous ne

122 pouvons permettre à personne de toucher à la religion islamique

123 que nous confessons tous. Mais il nous faut cerner le différend

124 dans un cadre naturel. Et moi, en tant que l'un d'entre vous qui

125 prend le pouls de la rue égyptienne, je sais que ce sujet est un

126 sujet qui revêt de l'importance pour le public. Et nous respectons

127 les opinions du public. Et nous, ici, nous respectons

128 les principes auxquels nous enjoint la vraie religion. Mais je

129 veux attirer l'attention [sur le fait que] ce sujet est un sujet de

130 grande divergence entre les jurisconsultes et les imams. Nous

131 avons effectivement entendu ces...

132 (Vacarme et interruptions de certains députés et des voix disant:

133 Non, non, il n'y a pas de divergence sur le voile, au contraire il y

134 a consensus à son sujet).

Le président de l'Assemblée

135 Merci, le temps qui vous est alloué est terminé à présent. Je prie

136 Monsieur l'honorable député 'Alâ' 'Awâjah, de [nous faire part de

137 ses] commentaires.

Quatre interruptions jalonnent cet extrait. La première (lignes 86-88) intervient dans un moment de transition entre deux tours de parole (intertours). L'intervenant, rompant l'ordre normal de passage de la parole, saisit l'opportunité d'un entre-deux pour insérer des propos. Leur formulation permet d'observer la grammaire de verbes tels que «je n'imaginais pas» (lam akun atasawwar) et "je sais » (a lam). Le premier, utilisé dans une forme négative, est un acte de langage permettant contextuellement de formuler un commentaire de surprise (à la lecture par le président de l'Assemblée de la lettre envoyée par le ministre de la Culture: "Je n'imaginais pas que votre excellence lirait à haute voix cette missive »). Le second verbe, quant à lui, performe, dans ce contexte précis, l'induction d'un mécanisme de solidarité négative (faire état d'un savoir quant à l'opinion privée d'autrui rendant difficile voire impossible à celui-ci de s'en dédire : " et je sais que vous n'en êtes pas convaincu »). Le président parvient à se désengager de l'alignement forcé que vise l'usage de ce verbe constatif en faisant glisser la question sur le terrain, non pas de ses convictions, mais des 
règles de procédure à respecter. À nouveau, on peut observer comment le Règlement intérieur, loin de n'être qu'un ensemble de normes procédurales dictant le déroulement de la séance, est avant tout une ressource aux mains des membres de l'Assemblée dont ils usent aux fins pratiques, interactionnelles, argumentatives et contextuelles qui émergent au fur et à mesure de la progression du débat. La deuxième interruption (lignes 100102) intervient pour sa part à l'intérieur même d'un tour de parole (intratours). Elle est faite de deux interventions successives qui saisissent l'un des derniers mots prononcés par l'orateur légitime (« des opinions qui doivent ») pour les préciser de manière contrastive ( $"$ une opinion contre la religion »; " une opinion fausse »). Alors que l'orateur légitime cherche à faire valoir la nécessité de respecter la pluralité des opinions, les intervenants illégitimes cherchent à reformuler le propos de manière à empêcher la mise en équivalence des différents points de vue et à imposer autoritairement un régime d'interprétation unique. La troisième interruption (lignes 116-117) prend, elle, la forme d'une question rhétorique. La tentative de l'orateur de minimiser l'importance de la déclaration du ministre est contrée par une intervention qui dénie la possibilité de détourner le débat en cours et contient une menace voilée. On peut parler ici d'une technique consistant à maximiser, inversement et négativement, les effets d'une minimisation. L'intervention se présente comme une question (ligne 117 : « Défendez-vous les erreurs ?! »), mais c'est une question d'un point de vue purement syntaxique et rhétorique, qui feint l'interrogation pour mieux porter une accusation et, en conséquence, faire peser sur l'orateur la menace du discrédit. En ce sens, cette question est implicative (si vous minimisez l'affaire, vous cherchez en fait à défendre des erreurs) et conséquentielle (si vous défendez des erreurs, vous êtes passible des mêmes accusations que le ministre).

La dernière interruption de cet extrait (lignes 132-134) consiste dans le déni d'une solidarité sans consensus et dans l'affirmation d'une solidarité consensuelle. Alors que l'intervenant tendait à faire valoir la pluralité des interprétations sur la question du voile (lignes 128-130 : «Et nous, ici, nous respectons les principes auxquels nous enjoint la Vraie Religion. Mais je veux attirer l'attention [sur le fait que] ce sujet est un sujet de grande divergence entre les jurisconsultes et les imams »), une voix non identifiée prétend à l'unanimité des avis sur le sujet (lignes 133-134: " Non, non, il n'y a pas de divergence sur le voile, au contraire il y a consensus à son sujet »). L'affirmation de ce consensus ne repose sur aucun autre argument que lui-même, il est en quelque sorte performatif: il y a consensus parce qu'on en affirme autoritairement l'existence et qu'il n'existe personne qui 
soit en mesure d'en donner la preuve contraire, non pas pour cause d'un manque d'arguments, mais en raison d'un contexte qui en empêche l'énonciation. C'est sur cette interruption que s'achève le tour de parole de l'orateur. Alors que, précédemment, le président de l'Assemblée avait corrigé l'atteinte faite au déroulement normal du débat parlementaire en rappelant à l'ordre la personne qui en avait perturbé le cours, au motif de l'existence de règles de procédure qu'il conviendrait de respecter, il saisit cette fois l'occasion qui lui est offerte par cette interruption pour couper la parole à l'orateur et l'allouer à l'orateur suivant. C'est encore à l'aide du Règlement intérieur, auquel il fait référence de manière implicite, que le président agit, quand il prétexte du dépassement du temps de parole (ligne 135 : «le temps qui vous est alloué est terminé à présent »). Il s'agit en fait d'une nouvelle forme de réparation à une interruption, une réparation qui toutefois sanctionne l'orateur et non la personne qui l'a interrompu. D'une certaine façon, le président indexe l'interruption sur le propos de l'orateur, dans la mesure où c'est la teneur de celui-ci qui l'explique et même la justifie. Les propos du président rendent cette interruption, bien que réglementairement anormale, substantiellement légitime et font porter la responsabilité de l'entorse au cours normal du débat, en amont de la chaîne de causalité, sur la personne dont les propos ont occasionné l'infraction. En coupant la parole à l'orateur, le président ne fait qu'exercer son rôle procédural, mais, ce faisant, il laisse aussi entrevoir son positionnement dans le débat.

Les interruptions que nous venons de passer en revue constituent des tours de parole à part entière qui donnent au débat une dimension interactive. L'ensemble des règles procédurales organisant les débats parlementaires visent, pas seulement en Égypte, à réduire au maximum la dimension dialogique de ceux-ci, de telle sorte qu'ils prennent généralement la forme de juxtapositions monologiques ${ }^{13}$. En ce sens, les interventions ne se répondent pas les unes aux autres, mais se succèdent sans relation immédiate. Elles sont toutefois reliées par la thématique générale du débat. En outre, elles peuvent parfois interagir avec un temps de décalage (le temps séparant les tours de parole initialement prévus et un tour de parole légitime visant à répondre à un tour antérieur). À l'inverse, les interruptions, bien qu'elles ne soient pas réglementaires et probablement parce qu'elles ne le sont pas, sont directement dialogiques. Elles constituent la deuxième partie d'une paire adjacente et séquentielle spécifique que le tour de parole de l'orateur légitime (qui en est la première partie) conditionne et rend

\footnotetext{
${ }^{13} \mathrm{Cf}$. au sujet des interruptions BEVITORI, 2004.
} 
contextuellement pertinente. Là où le débat parlementaire fonctionne normalement de manière contiguë mais déconnectée, l'interruption procède, pour sa part, de manière contiguë et immédiatement connectée. On notera enfin que les interruptions peuvent avoir une incidence sur le cours séquentiel du débat, mais pas nécessairement. C'est ainsi que, comme on l'a $\mathrm{vu}$, la quatrième interruption de l'Extrait 12 permet au président de l'Assemblée d'allouer la parole à l'orateur suivant, réalisant par là-même une double opération : couper court au tour de parole en cours et rétablir l'ordre normal du débat. Inversement, une interruption comme celle reproduite à l'Extrait 13, qui consiste en une amplification ironique (lignes 837-838) des propos de l'orateur légitime (ligne 834), ne modifie pas le cours séquentiel du débat et ne semble pas justifier de réparation de la part du président de l'Assemblée : l'intervention de l'orateur se poursuit comme si de rien n'était.

Extrait 13 : séance parlementaire du 20 novembre 2006 (retranscription verbatim)

A.M.M. Darwîsh (Ahmad Abû Hajjî)

827 Pour commencer, Monsieur le président, le ministre a dit trois

828 vérités, dans le mémoire qu'il vous a envoyé. La première chose

829 qu'il a dite, c'est que sa culture n'est pas notre culture, et c'est

830 une vérité. Il a dit qu'il ne s'y entend pas en matière religieuse,

831 et c'est une vérité. Et il a dit qu'il est malade, et nous savons que

832 le malade... Ce sont les trois vérités qu'il a mentionnées dans le

833 mémoire qui se trouve devant vous.

834 (voix de Monsieur l'honorable député Rajab Hilâl Hamîda: De

835 quelle maladie souffre le ministre ?)

M. l'hon. Député A.M.M. Darwîsh (Ahmad Abû Hajîî)

836 J'espérais qu'un ministre responsable dans notre gouvernement

837 se présente devant l'Assemblée et ses députés, mais j'ai le

838 sentiment que Monsieur Fârûq Husnî n'a pas le courage

839 suffisant de venir et de faire face aux députés [...]

L'ensemble des remarques formulées précédemment au sujet des interruptions vaut pour cette forme spécifique d'alignement pragmatique sur les propos de l'orateur légitime que sont les applaudissements. Dans la grammaire pratique du débat parlementaire, ceux-ci se situent dans la contiguité immédiate des propos qu'ils viennent acclamer. Ils sont la deuxième partie d'une paire adjacente et séquentielle dont la première partie, les propos de l'orateur, conditionne contextuellement la survenance. Dans le 
débat de l'Assemblée du Peuple, on en retrouve plusieurs occurrences (16, pour être exact). La notation des applaudissements - qui apparaît sur l'original de la retranscription verbatim - permet d'identifier très exactement la partie du discours sur laquelle ils se greffent.

Dans leur article sur les applaudissements dans les meetings politiques, Heritage et Greatbatch ${ }^{14}$, s'appuyant sur le travail d'Atkinson ${ }^{15}$, identifient des moyens sémantiques et pragmatiques propres à susciter l'adhésion positive du public. Sémantiquement, la formulation d'une critique à l'égard d'une cible déterminée, autour de laquelle l'orateur s'attache à créer le consensus stigmatisant, fonctionne de manière souvent efficace. Pragmatiquement, ils identifient sept configurations rhétoriques susceptibles d'induire l'ovation du public : les contrastes, les listes de trois, la solution à une énigme (puzzle-solution), en-tête et ligne de force (headline et punch line), combinaison, prise de position (position taking) simple ou complexe, poursuite (pursuit) simple ou complexe. Dans le débat de l'Assemblée du Peuple, plusieurs de ces cas de figure se retrouvent. Au niveau sémantique, tout d'abord, où l'on a pu déjà observer comment la demande de démission du ministre s'était établie comme une sorte de refrain régulièrement suivi des applaudissements d'une partie de l'Assemblée :

Extrait 14 : séance parlementaire du 20 novembre 2006 (retranscription verbatim)

Le président de l'Assemblée

510 Je prie Madame l'honorable députée Hiyâm 'Âmir de [nous faire

511 part de ses] commentaires.

Mme l'hon. Députée H. 'A. 'A. 'U. 'Âmir

512 Au nom de Dieu le Clément le Miséricordieux

513 La prière et la paix sur le plus noble des envoyés. En mon nom

514 et au nom de toute femme en Égypte et dans le monde arabe, je

515 dis au ministre qui a tenu ce propos, ce n'est pas un propos

516 correct et il n'a aucune relation avec l'islam. Je réclame la

517 démission du ministre parce qu'il ne mérite pas d'être ministre

518 ou toute autre chose (applaudissements). Merci Monsieur le

519 président.

${ }^{14} \mathrm{HERITAGE}$ et GREATBATCH, 1986.

${ }^{15}$ ATKINSON, 1984. 
Au niveau pragmatique, ensuite, où plusieurs des cas de figure se retrouvent. C'est ainsi que, dans ce même Extrait 14, le fait que l'orateur légitime soit une femme voilée, ayant déjà marqué sa position en interrompant la séance à plusieurs reprises, qui soit au nombre de ces responsables féminins portant le voile identifiées à d'autres occasions (cf. Extrait 16), apparaît comme l'affirmation préalable d'un état de fait dont découle, nécessairement, la prise de position ultérieure consistant à demander la démission du ministre.

Les applaudissements à l'extrait suivant semblent intervenir par rapport à une partie déterminée du discours. Ils font suite à une sorte d'énigme posée par l'orateur (orientation personnelle ou orientation du gouvernement ?) à laquelle il entend apporter une réponse (orientation personnelle) en montrant que la majorité des Égyptiennes, y compris les femmes de sa famille, c'est-àdire les femmes de la famille d'un député du parti au pouvoir, portent le voile. Il s'agit d'une forme d'intervention syllogistique, où la majeure est faite de la représentativité du gouvernement, la mineure, du port du voile chez une série de femmes liées à ce gouvernement et la conclusion, de ce que le gouvernement ne peut être contre le voile. Il en résulte donc bien que les déclarations de Fârûq Husnî expriment une position personnelle et non une orientation du régime.

Extrait 15 : séance parlementaire du 20 novembre 2006 (retranscription verbatim)

M. l'hon. Député K. al-Shadhlî

905 [...] et c'est ce qui m'a poussé à intervenir : Est-

906 ce que ces propos sont l'orientation du gouvernement ou une

907 orientation de régime? C'est un sujet sur lequel je dois

908 répondre. Par principe, je parle comme député. Je n'ai pas le

909 sentiment que ce soit une orientation générale. Pourquoi ? Parce

910 que l'orientation générale, mes frères, c'est que la plupart de nos

911 parents, parmi les femmes, sont voilées, et les filles sont une très

912 grande proportion d'entre elles. Le peuple égyptien porte le voile

913 et moi, personnellement, ma fille et mon épouse sont voilées

914 (applaudissements). [...]

La liste de trois constitue une autre technique pragmatique d'initiation d'applaudissements. Elle consiste, d'une part, en l'accentuation du propos par l'établissement d'une liste d'exemples de personnes qui, à suivre les propos de Fârûq Husnî, tomberaient dans la catégorie des "attardés 》 (Extrait 16, lignes 321-324: « la ministre », « la députée », « la docteure »). 
D'autre part, la technique de la liste consiste en une énumération dont la complétion, conventionnellement arrêtée à trois items, peut être anticipée par l'audience.

Extrait 16 : séance parlementaire du 20 novembre 2006 (retranscription verbatim)

M. l'hon. député M. Mustaphâ Bakrî

320 Deuxièmement : quelle sera la position du ministre quand il entrera au

321 Conseil des ministres et qu'il verra Madame 'Â'isha 'Abd al-Hâdi,

322 ministre de la main d'œuvre, alors qu'elle se couvre la tête. La ministre

323 est-elle attardée ? Madame la députée est-elle attardée ? La Dr.

324 Yamna al-Hamâqî est-elle attardée ? (applaudissements)

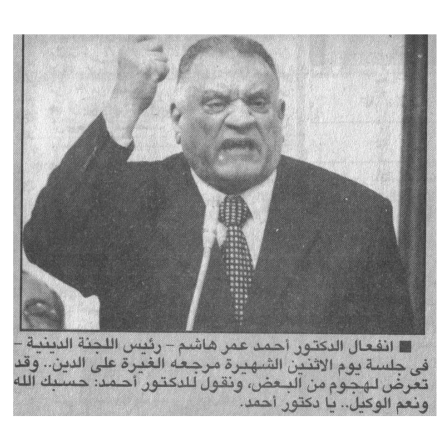

Le député Hâshim lors de son intervention $(a l-U s b \hat{u} ; 25$ novembre 2006)

Le contraste constitue une dernière technique pragmatique d'initiation d'applaudissements. Il s'agit pour l'orateur, dans ce cas, d'accentuer la différence entre une valeur positive et une valeur négative. À l'Extrait 17, la défense de l'islam (nous autres gardiens des valeurs de l'islam) se présente en contrepoint des attaques dont celui-ci fait l'objet (eux les diffamateurs et travestisseurs). Dans ces catégorisations d'appartenance contrastant un "nous" vertueux à un «eux» malfaisant, l'effet rhétorique est obtenu par le brouillage de la ligne de partage : les déclarations sont le fait de «l'un d'entre nous». (lignes 1001-1002). C'est à l'implication de ce brouillage (l'un d'entre nous est en fait l'un d'entre eux) que les députés réagissent en applaudissant :

Extrait 17 : séance parlementaire du 20 novembre 2006 (retranscription verbatim)

\section{M. l'hon. Député A. 'A Hâshim}

997 [...], alors qu'est sorti un livre qui diffame la demeure de la

998 prophétie (bayt al-nubuwwa), intitulé Les Versets sataniques, et le 999 phénomène du travestissement du Coran et autres images 1000 malfaisantes et infâmes. Ces déclarations ne nous viennent pas 
1001 seulement du Vatican, pire, elles viennent de nous autres, 1002 malheureusement, des déclarations malfaisantes (applaudissements)

\section{Pertinences et audiences}

Le site dialogique de l'Assemblée du Peuple amène les députés, d'une manière institutionnellement contrainte, à mobiliser des registres de pertinence et à s'adresser à des audiences multiples ${ }^{16}$. Un registre de pertinence peut se définir comme un répertoire sur lequel s'opère un alignement discursif et dont on revendique l'observance, y compris les principes qui relèvent de l'ordre de vérité qu'il établit ${ }^{17}$. Dans le débat portant sur " l'affaire Fârûq Husnî », différents registres de pertinence sont utilisés. Le fait que la séance soit inaugurée au nom de Dieu établit d'emblée la légitimité du recours à la pertinence islamique. Qu'elle le soit aussi, légalement, au nom du peuple garantit parallèlement la validité de la pertinence démocratique et constitutionnelle, c'est-à-dire du mécanisme de représentation parlementaire garanti formellement par la loi fondamentale et les principes de l'État de droit. Les pertinences ne sont cependant jamais données une fois pour toutes, elles demeurent toujours émergentes. En effet, la seule invocation de la légitimité d'un registre de pertinence ne suffit pas à en assurer l'actualité ; il convient encore qu'il fasse l'objet d'une actualisation. Toujours dans ce débat, ce sont également des pertinences catégorielles qui trouvent à s'afficher, qu'il s'agisse de l'identité égyptienne ou du nationalisme arabe. Ainsi, les registres de pertinence sont-ils multiples, imbriqués, modulables. Seule l'étude de leur déploiement contextuel permet d'en mesurer l'importance et d'en observer le mécanisme.

Extrait 18 : séance parlementaire du 20 novembre 2006 (retranscription ${\text { verbatim })^{18}}^{18}$

M. l'hon. Député 'A.D.I.'Abd al-Ghânî ('Alâ' 'Awajah)

\footnotetext{
${ }^{16}$ Ces audiences ont accès aux débats par le biais de leur retransmission (éventuelle) sur la chaîne hertzienne d'Etat, par leur retranscription accessible sur le site du Parlement ou reproduite dans la presse, et par la narration que des témoins directs ou indirects peuvent en faire.

${ }^{17}$ FERRIÉ, DUPRET, LEGRAND, 2008

18 'Âtif Sidqî fut Premier ministre de 1986 à 1996. Fârûq Husnî est ministre de la Culture depuis 1987.
} 
138 Monsieur le président ceci est un sujet de la plus haute importance et

139 il faut que nous nous en préoccupions tous. Oui, nous sommes un

140 État islamique. Oui, notre constitution stipule que la sharî'a est la 141 source principale de la législation. Oui, nous sommes tous 142 musulmans. Oui, nous tenons tous à la religion islamique, à la 143 prédication $\left(d a^{\prime} w a ̂\right)$ islamique et aux principes de l'islam. Pour nous 144 tous, notre relation à Dieu - qu'Il soit loué et exalté ! - est la relation 145 de tout individu musulman zélé (ghuyûr) à sa religion.

Cet extrait manifeste l'étroite articulation des pertinences islamique et constitutionnelle. L'affirmation de ce que l'Égypte est un État islamique (ligne 140) renvoie aussi bien à une appartenance religieuse qu'à une disposition de la Constitution. Cette même Constitution stipule, à son article 2, que la Loi islamique ( $\operatorname{sharî̀}^{\prime} a$ ) est la source principale de la législation, ce que l'orateur exploite pour souligner l'inscription du droit dans le cadre islamique. Le propre de la mobilisation d'un registre de pertinence est de projeter à l'avant-plan les termes dans lesquels le débat va pouvoir se déployer, tant et si bien qu'il devient impossible d'y prendre part autrement qu'en recourant aux ressources qu'il offre. Que ce soit de manière consensuelle, pour marquer un désaccord ou par défaut, les termes du débat sont établis et il devient impossible d'y échapper. Cette projection à l'avantplan recourt elle-même à diverses techniques rhétoriques, tels, à l'Extrait 26, la scansion (lignes 139-143 : "Oui, nous... ») et la liste (lignes 142-143 : la religion islamique, la prédication islamique, les principes de l'islam).

Les pertinences démocratique (qui s'articule au principe majoritaire) et constitutionnelle (qui s'articule au principe de légalité) constituent assurément un registre de choix sous la coupole de l'Assemblée du Peuple :

Extrait 19 : séance parlementaire du 20 novembre 2006 (retranscription verbatim)

M. l'hon. Député M.'A.H. Dâwud

$692[\ldots]$ c'est l'Assemblée du Peuple que le peuple a choisie

693 et c'est le ministre qui nous vient de l'époque du gouvernement

694 de 'Âtif Sidqî. Chaque Premier ministre qui est venu se

695 retrouvait avec un ministre imposé, Fârûq Husnî, celui qui peut

696 aujourd'hui mépriser la volonté du peuple d'Égypte, qui est la

697 terre d'al-Azhar, qui est la source de l'islam, qui est le phare de

698 l'islam. Aujourd'hui, nous nous levons pour protester contre

699 l'atteinte au voile en France ou dans n'importe quel pays. 
700 Aujourd'hui, que ferons-nous, Monsieur le président, comme 701 peuple d'Égypte, et qui répondra aux députés de l'Assemblée du 702 Peuple égyptien, de la majorité et de l'opposition ? Parce qu'il y 703 a une atteinte aux fondements de la religion, Monsieur le 704 président. C'est ce qu'a dit le Dieu glorifié, le Très-Haut, ce 705 n'est pas Ahmad Nazîf qui l'a dit, non, Monsieur le président, on 706 ne peut pas laisser passer ce sujet avec indifférence, sinon 707 l'islam sera un objet de moquerie pour le gouvernement du Parti 708 National. En même temps, je remercie mes collègues, les 709 députés du Parti National, les députés de l'opposition et les 710 indépendants, parce qu'il y a une position commune contre un 711 ministre qui a porté atteinte à la religion vraie.

712 Monsieur le président, je suis prêt au martyr sur le chemin de la 713 religion et je suis prêt au martyr sur le chemin de la Nation, mais 714 je ne suis pas prêt au martyr sur le chemin...*. Merci Monsieur 715 le président.

* À l'endroit des points, des propos qu'il a été décidé de supprimer.

Cette intervention est l'une des prises de position les plus explicitement politiques de la séance. Il n'est pas indifférent de savoir que son auteur est membre de l'un des partis d'opposition ultra-minoritaires de l'Assemblée du Peuple (Néo-Wafd). Son argumentaire consiste à établir un contraste entre les représentants de la Nation élus à l'Assemblée, issue de la volonté populaire (ligne 692), et Fârûq Husnî, membre inamovible du gouvernement égyptien depuis une vingtaine d'années (lignes 693-694). À partir de ce contraste, l'orateur peut faire ressortir le caractère imposé de la présence du ministre de la Culture et son indifférence à la volonté populaire (lignes 694696). La volonté populaire est alors mise en équivalence de la nature islamique de l'Égypte, laquelle est soulignée par une liste de trois (lignes 698-699). L'orateur crée ici un deuxième contraste entre le gouvernement qui, si le Parlement n'y prête garde, pourrait en finir par se moquer de l'islam (lignes 706-708), et les députés, toutes tendances confondues, qui font barrage aux atteintes à "la religion vraie" (lignes 710-711). En conclusion, le député procède à une dernière liste de trois où le martyr pour l'islam ou la Nation (lignes 712-713) est opposé au martyr pour une vision perverse de la société (ligne 714). L'ensemble de l'intervention de M. 'A. 'A. Dâwud apparaît ainsi organisée autour de l'idée, d'une part, de la légitimité démocratique de l'Assemblée du Peuple qui, émanation de la volonté populaire, ne peut qu'être fidèle à la conception «vraie » de la religion et constitue, partant, l'ultime gardien de son respect et, d'autre part, 
l'illégitimité des gouvernements successifs auxquels le ministre de la Culture est imposé, dépourvus de représentativité populaire et donc toujours susceptibles de tourner la religion en dérision. Le registre de pertinence démocratique permet d'établir une ligne de partage entre la représentativité, émanant du principe électif, et l'autoritarisme, et son association au registre de pertinence islamique lui permet de mettre en équation démocratie et islamité authentique.

Ce même extrait exprime également l'orientation de l'orateur vers des audiences multiples (majorité, opposition, indépendants). La projection des destinataires d'une intervention autorise la convocation d'actants, matériels ou virtuels, permettant d'incarner les pertinences. Dans un jeu imbriquant registres de pertinence et audiences, les députés catégorisent les publics qu'ils représentent et auxquels ils s'adressent pour mieux démarquer les camps en présence, créer les antagonismes et les alliances et provoquer les alignements. Cette stratégie rhétorique est fondée sur l'idée que le discours possède effectivement une capacité performative.

Extrait 20 : séance parlementaire du 20 novembre 2006 (retranscription verbatim)

Le président de l'Assemblée

573 Je prie Monsieur l'honorable député 'Abd al-Rahîm al-Ghûl de

574 [nous faire part de ses] commentaires.

M. l'hon. Député 'A.R. al-Ghûl

575 Au nom de Dieu le Clément le Miséricordieux.

576 La prière et la paix sur le plus noble des envoyés, notre seigneur

577 Muhammad, sur sa famille et ses compagnons, et le salut.

578 Monsieur le docteur Ahmad Fathî Surûr, président de

579 l'Assemblée.

580 Quoi de plus beau que [de voir] l'opposition, la majorité et les

581 indépendants être d'accord, si bien que notre ferveur pour notre

582 religion, personne ne la partage. L'islam est ce qui réside dans le

583 cœur et qu'atteste l'action. C'est pour cela que je crois que celui

584 qui émet une opinion en cela, ce peut être le cheikh al-Azhar ou

585 n'importe quelle personnalité de nos cheikhs distingués, mais

586 que le ministre de la Culture a dépassé les bornes et a parlé d'[un

587 sujet] qui ne le concerne pas. Il ne fait pas de doute que nous

588 autres, au Parti National Démocratique, au nom de tout le peuple

589 d'Égypte, nous ne condamnons pas seulement les propos de

590 Monsieur le ministre, nous nous élevons contre lui et nous 
591 sommes d'accord avec l'opposition, les indépendants et la 592 majorité (applaudissements) sur le fait qu'il a dépassé les bornes. 593 Je crois dans les propos de Monsieur le docteur Zakariyâ 'Azmî, 594 quand il a parlé et a dit que ce ministre exprimait son opinion 595 personnelle et n'exprimait pas l'opinion du gouvernement ou 596 l'opinion du régime.

597 Il ne fait pas de doute que nous autres, sous la coupole de 598 l'Assemblée, nous disons que, si ce ministre exprimait donc son 599 opinion, il lui faut retirer cette opinion, parce que l'Égypte est un 600 État islamique. Dès lors, nous avons exprimé notre opposition 601 aux États étrangers qui ne veulent pas que qui que ce soit en 602 parle; dès lors, nous avons exprimé notre opposition aux États 603 européens qui ne veulent pas du voile. Le rôle de l'Égypte [se 604 manifeste] en tant que pays musulman. Nous avons pleinement 605 foi et nous n'accepterons jamais, de près ou de loin, que 606 n'importe qui touche à notre religion, qu'il appartienne au 607 pouvoir exécutif ou qu'il appartienne au Parti.

608 Dès lors, l'opposition, la majorité et les indépendants sont 609 d'accord, parce que nous sommes tous croyants en notre 610 Seigneur Dieu et en Muhammad, [Son] Prophète et Envoyé. 611 Merci Monsieur le président.

Plusieurs audiences sont convoquées: le président de l'Assemblée, les différents groupes de députés, le Parti National Démocratique, le peuple égyptien, un député précis, les États étrangers. C'est de manière formelle et routinière que la parole est adressée au président de l'Assemblée, ce qui le constitue en audience procédurale. Il convient toutefois de noter que c'est avec une certaine emphase que l'orateur y procède, ce qui laisse entendre qu'il n'est pas indifférent d'en obtenir le ralliement. Les députés de la majorité, les députés de l'opposition et les députés indépendants constituent des publics également présents physiquement. En leur adressant la parole directement, en tant que l'un d'entre eux, et en soulignant la convergence de leurs points de vue, l'intervenant projette l'existence d'une solidarité avec consensus sur la primauté et l'intangibilité de l'islam. Le jeu des audiences lui permet de transcender les oppositions parlementaires classiques pour produire un " nous » dont aucun participant ne peut se dédire (lignes 581582). Ce «nous », qui semble être ensuite réduit au seul PND (ligne 588) est en fait mis en équivalence avec l'ensemble du peuple égyptien (ligne 589) et avec toutes les forces parlementaires (ligne 591-592) pour projeter, une 
deuxième fois, une solidarité avec consensus qui, alliant, dans les termes de Heritage et Greatbatch ${ }^{19}$, poursuite d'un thème énoncé abordé précédemment dans son intervention (l'alliance de la majorité, de l'opposition et des indépendants) et liste de trois contrastive (le martyr pour l'islam et pour la nation, mais pas pour le ministre), provoque des applaudissements (ligne 592). La voix d'un député précis, Zakariyâ 'Azmî, figure importante du PND, est également incorporée intertextuellement dans cette intervention, transformant cette figure, du simple fait de sa présence dans l'hémicycle et de cette convocation, en une audience dont le ralliement, comme c'était le cas avec le président de l'Assemblée, lui aussi cacique du parti, n'est pas indifférent. On remarque ainsi comment la sollicitation explicite d'une audience personnalisée procède, pour 'A.R. al-Ghûl, député PND rallié, de son inscription dans la politique interne du parti majoritaire et des mécanismes d'alliances qui l'accompagnent. Dernier cas de figure, dans cet extrait, du jeu des audiences et de leur imbrication avec les registres de pertinence, la référence aux États étrangers et européens intervient comme une sorte de mise en abîme : l'audience étrangère, à laquelle l'Égypte s'est adressée pour marquer son opposition, donne l'occasion à l'orateur d'établir une dichotomie entre le «nous » égyptien et musulman et le « eux » étranger et non musulman, posant les bases d'un renvoi de la personne du ministre dans la deuxième catégorie. Les registres de pertinence islamique et national mobilisent, aux fins pratiques et immédiates du débat parlementaire en cours et par le truchement de catégorisations d'appartenance, l'usage d'audiences contrastées qui avait été fait précédemment.

Extrait 21 : séance parlementaire du 20 novembre 2006 (retranscription verbatim)

M.H. Baghdâdî F.

456 Monsieur le professeur docteur Président de l'Assemblée,

457 Messieurs les députés: Cette déclaration scandaleuse

458 (mushayyin) qu'a faite Monsieur le professeur Fârûq Husnî agite

459 l'opinion publique arabe, égyptienne et islamique. Cette

460 déclaration contredit les enseignements de la sharî' $a$ islamique

461 et ses fondements. En tant que représentant du gouvernement, il

462 ne fallait pas qu'il s'immisce dans les affaires religieuses qui ont

463 leurs statuts (ahkâm) et leurs savants (fuqahâ'). Au verset 31 de

464 la sourate La Lumière, le Glorieux et Très-Haut dit : "Et dis aux

465 croyantes qu'elles baissent leurs regards, qu'elles préservent leur

\footnotetext{
${ }^{19}$ HERITAGE \& GREATBATCH, 1986.
} 
466 chasteté, qu'elles ne montrent pas leurs atours sinon ce qu'il en 467 apparait, qu'elles rabattent leur voile (khumur) sur leur poitrine 468 et qu'elles ne montrent leurs atours qu'à leurs maris ». Parole du 469 Dieu Puissant.

470 Dès lors, cela fait partie des fondements du Coran et des 471 fondements de la noble Tradition. C'est ce qui a été révélé dans 472 notre Saint Coran. Nous sommes les représentants de l'instance 473 parlementaire du Parti National. Nous disons que ce ministre ne 474 mérite pas d'être membre du gouvernement du Président 475 Muhammad Husnî Mubârak (applaudissements). Également, 476 j'exige qu'il présente sa démission ou qu'il s'excuse et baisse la 477 tête devant le peuple égyptien pour ce qui lui a échappé dans 478 cette déclaration. Merci.

L'intervention de M.H. Baghdâdî se présente de manière comparable à celle reproduite à l'extrait précédent : des audiences multiples (le président, les députés, l'opinion publique, les représentants du PND, le gouvernement, le président Mubârak, le peuple égyptien) et des registres de pertinence variés (le registre des appartenances arabe, égyptienne, islamique ; le registre de l'islam, du Coran et de la sharî' $a$; le registre des institutions politiques). Elle se caractérise en même temps par une structure à la fois descriptive, normative et conséquentielle : le député, dans un premier temps, formule un jugement (lignes 457 : " scandaleuse »; ligne 462: " ne fallait pas qu'il s'immisce 》) ; dans une deuxième temps, il pose l'arrière-plan factuel (lignes 458-461: " agite l'opinion» et «contredit les enseignements de la sharî'a »), référentiel (lignes 460-472: la sharî'a, le Coran, la Tradition) et textuel (lignes 464-468: sourate La Lumière) de ce jugement; dans un troisième temps, établit le statut lui permettant de le formuler et, dans un dernier temps, en tire les conséquences motivées par l'arrière-plan, en se fondant sur sa position statutaire. Le collectif " nous " par lequel l'orateur s'associe au groupe parlementaire PND à l'Assemblée pour juger de l'indignité du ministre est suivi d'un "je», individuel, par lequel il radicalise l'injonction (ligne 476: "j'exige ») faite à Fârûq Husnî de démissionner ou de s'excuser. On remarquera l'alternative laissée par ces derniers propos entre la démission et les excuses, là où d'autres interventions concluaient à la nécessité cumulative des deux. 


\section{Remarques conclusives}

Les différentes interventions à l'Assemblée du Peuple sont l'occasion d'observer le discours politique en contexte et en action. Le discours parlementaire est contraint, dès lors qu'il s'inscrit dans une procédure normée par des textes (la Constitution et le Règlement intérieur) et des pratiques (les modes propres à l'Assemblée du Peuple d'allouer la parole, d'autoriser des infractions au Règlement intérieur, d'interrompre, d'ignorer, etc.). Mais la contrainte procédurale et institutionnelle ne doit pas seulement être considérée sous l'angle de ses effets restrictifs; elle offre aussi des interstices, des ressources dont les participants à l'interaction parlementaire usent aux fins pratiques de leur action, entre autres politique.

Dans cette étude, nous avons pu observer comment la séquence, bien que partiellement pré-formatée, était aussi façonnée par les interventions et interruptions des différents participants. Les tours de parole légitimes sont effectivement pré-alloués, mais leur taille et leur nature peut se trouver profondément altérée par l'interaction avec les autres membres de l'Assemblée. En outre, le président exerce une fonction d'ordre, qui établit, alloue et accorde les tours de parole, mais son rôle va bien au-delà, dès lors qu'il intervient de multiples façons pour couper court à certains propos, les circonscrire, les corriger, expliquer des règles de procédure (mais parfois aussi des règles substantielles), étayer des prises de position. Ce faisant, loin de n'exercer qu'un rôle procédural, le président intervient directement pour donner au débat une forme et un contenu. Les différents types d'interruption, qu'il s'agisse de prises de parole illégitimes, de vacarme ou d'applaudissements, contribuent, pour leur part, à donner au débat un tour interactif et lui impriment à l'occasion une direction nouvelle. Ils permettent aussi de marquer et de provoquer des alignements et des oppositions (entre autres catégoriels) entre des participants que le respect de la règle procédurale aurait autrement contraint à intervenir par blocs discursifs juxtaposés et relativement indifférents les uns aux autres. Quant à l'orientation vers de multiples audiences, présentes et absentes, matérielles et virtuelles, elle permet aux intervenants de poser les termes du débat d'une manière sensible à ses destinataires présumés. La convocation de ces publics contribue elle aussi à créer des alignements et des oppositions et donc à non seulement fixer les termes autorisés du débat, mais également à en inférer une série de conséquences. La mobilisation de différents registres de pertinence, imbriqués les uns aux autres et étroitement liés aux diverses audiences sollicitées, permet enfin le déploiement de systèmes catégoriels déterminant les identités en présence, les gammes d'action interdites et 
autorisées, les légitimités, et l'ensemble des conséquences qui découlent nécessairement du recours à ces dispositifs de catégorisation.

Les modes de déploiement du débat parlementaire attestent de l'autonomie de ce site dialogique. En quelque sorte bouclée, autosuffisante, l'activité parlementaire s'appuie sur elle-même et renvoie à elle-même. Dans les termes de Teubner $^{20}$, elle est autopoiétique. En même temps, le débat parlementaire à propos de l'affaire Fârûq Husnî s'inscrit résolument dans un réseau dialogique : il trouve son origine et son déclenchement dans le réseau tel qu'il s'est développé et amplifié précédemment ; il en est d'une certaine manière l'une des extensions; il lui est rattaché par de nombreux connecteurs. Mais, outre que le site dialogique du Parlement puise dans le réseau antérieurement amorcé, il sert aussi de ressource à ses prolongements. Les propos des participants au débat parlementaire, leurs tours de parole, légitimes ou non, sont en effet susceptibles d'être repris par les médias, alimentant ainsi l'arborescence du réseau, entrant «en dialogue» avec ses différentes branches, participant, en un mot, à sa structure réticulaire.

${ }^{20}$ TEUBNER, 1993. 


\section{REFERENCES}

ATKINSON J. Maxwell and DREW Paul (1979) Order in Court: The Organization of Verbal Interaction in Judicial Settings, London: Macmillan

ATKINSON J. Maxwell (1984) Our Masters' Voices: The Language and Body Language of Politics, Londres: Routledge

BAYLEY Paul (éd.) (2004) Cross-Cultural Perspectives on Parliamentary Discourse, Amsterdam/Philadelphia: John Benjamins

BEVITORI Cinzia (2004) « Negotiating conflict: Interruptions in British and Italian parliamentary debates", in P. Bayley (éd.) Cross-Cultural Perspectives on Parliamentary Discourse, Amsterdam/Philadelphia: John Benjamins

BOVET Alain (2007) La Mise en question du génie génétique dans l'espace pubilc suisse: Analyse des pratiques médiatiques de mise en forme et de mise en cuvre du débat public, Thèse présentée à la Faculté des sciences économiques et sociales de l'Université de Fribourg (Suisse) [http://www.unifr.ch/sociomedia/ 2007/index.php?page=nouvelles-et-activites-14]

DUPRET Baudouin (2006) Le jugement en action : Ethnométhodologie du droit, de la morale et de la justice en Égypte, Genève : Droz

DUPRET Baudouin, BELHADJ Souhaill, FERRIÉ Jean-Noël (2007) « Démocratie, famille et procédure : Ethnométhodologie d'un débat parlementaire syrien» Revue européenne des sciences sociales, 139 : 5-44

DUPRET Baudouin, FERRIÉ Jean-Noël (2008) « Legislating at the shopfloor level: Background Knowledge and Relevant Context of Parliamentary Debates » Journal of Pragmatics, 40 : 960-978.

DUPRET Baudouin, KLAUS Enrique, FERRIÉ Jean-Noël (à paraître) « Scandale et réseau dialogique : ce que la morale fait au politique - A propos du foulard islamique au parlement égyptien »

FERRIÉ Jean-Noël, DUPRET Baudouin, LEGRAND Vincent (2008) « Retour sur la politique délibérative en action : Une position praxéologique » Revue Française de Science Politique

GREATBATCH David (1988) «A Turn-Taking System for British News Interviews » Language in Society 17(3)

HEATH Christian (1986) Body movement and speech in medical interaction, Cambridge: Cambridge University Press 
HERITAGE John (1998) « Conversation Analysis and Institutional Talk : Analysing Distinctive Turn-Taking Systems » in Cmejrková S., Hoffmannová J., Müllerová O. $\&$ Sveltá J. (éds.) Proceedings of the $6^{\text {th }}$ International Congress of IADA (International Association for Dialog Analysis), Tubingen: Niemeyer

HERITAGE, John \& GREATBATCH David (1986) «Generating Applause : a Study of Rhetoric and Response at Party Political Conferences » American Journal of Sociology 92

HERITAGE John \& GREATBATCH David (1991) « On the Institutional Character of Institutional Talk: The Case of News Interviews » in Boden D. \& Zimmerman D. H. (éds.) Talk and Social Structure, Berkeley: University of California Press

KLAUS Enrique, DUPRET Baudouin, FERRIÉ Jean-Noël (2008) «Derrière le voile : Analyse d'un réseau dialogique égyptien » Droit \& Société, 68 : XXX

LEUDAR Ivan \& NEKVAPIL Jiří (2007) « The war on terror and Muslim Britons' safety: A week in the life of a dialogical network », Ethnographic Studies, No 9 (Special issue: "Media, Wars and Identities: Broadcasting Islam, Muslims and the Middle-East”, Ed.: B. Dupret, J. Nekvapil, I. Leudar)

LEUDAR Ivan, MARSLAND Victoria \& NEKVAPIL Jiří (2004). «On membership categorization: 'us', 'them' and 'doing violence' in political discourse ", Discourse \& Society, Vol. 15 (2-3) : 243-266

LEUDAR Ivan \& NEKVAPIL Jiří (2004) "Media networks and political argumentation", Journal of Language and Politics $3: 2$

MCHOUL Alec (1978) « The Organization of Turns at Formal Talk in the Classroom », Language in Society 7

MEEHAN Albert J. (1997) «Record-Keeping Practices in the Policing of Juveniles », in Travers M. et Manzo J.F. (éds.) Law in Action: Ethnomethodological and Conversation Analytic Approaches to Law, Aldershot: Dartmouth/Ashgate

MEHAN Hugh (1985) « The Structure of Classroom Discourse » in Dijk T.A. (éd.) Handbook of Discourse Analysis Volume 3, New York: Academic Press

NEKVAPIL Jiři \& LEUDAR Ivan (2002) "On Dialogical Networks: Arguments about the Migration Law in Czech Mass Media in 1993" in Hester S. \& Housley W. (éds.) Language, Interaction and National Identity, Aldershot: Ashgate

SCHEGLOFF Emanuel A. (2007) Sequence Organization in Interaction. A Primer in Conversation Analysis 1, Cambridge: Cambridge University Press

TEUBNER G. 1993, Le droit, un système autopoiétique, Paris: PUF 